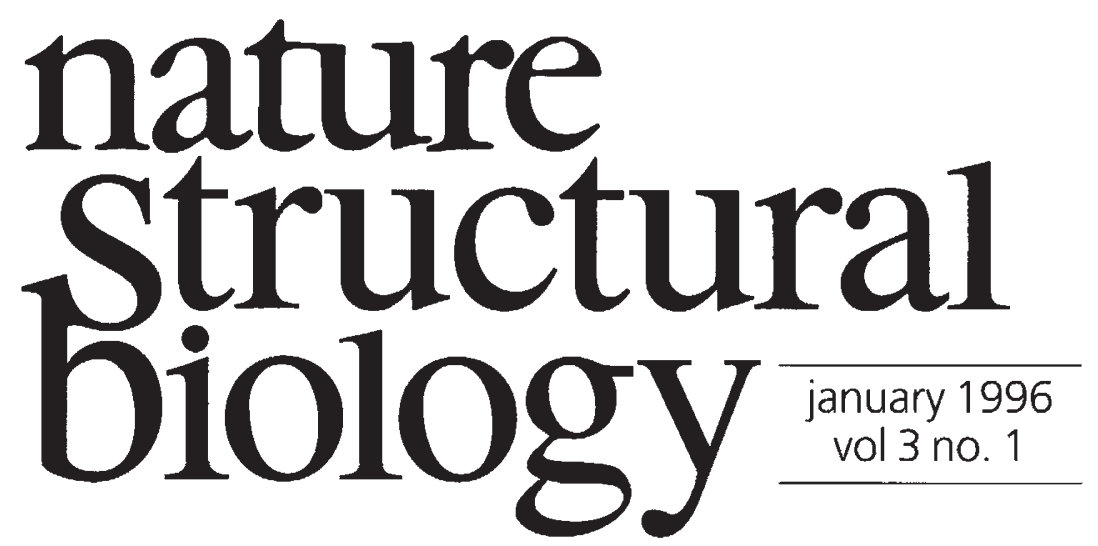

\title{
Coordinating coordinates
}

The issue of deposition of structure factors and, more particularly, atomic coordinates of published structures can be likened to the rose-it is both thorny and perennial. Continuing discussion of the topic ${ }^{1}$ illustrates the importance of the underlying issue: one of the basic tenets of the scientific method is freedom of information - researchers are required to make generally available both the methods and data that support their conclusions. And although progress has been made in providing generally accepted codes of behaviour within the field, the situation is still not perfect.

A paper published some fifteen years ago by Michael Rossmann and Patrick Argos $^{2}$ illustrates the persistence of the problem. The authors describe a method for deriving three-dimensional coordinates from stereo diagrams of published structures of biomolecules for which coordinates have not been deposited. As these authors point out, failure to deposit coordinates has led to the "unusual situation where scientists publicly present their results, but do not attempt to provide sufficient information for others to use quantitatively their 'published data".

Rossmann and Argos point out that authors may fail to deposit their coordinates for two reasons: (i) some structure determinations can take years of work and investigators may wish to analyse and digest their results so as to reap the benefit of their prolonged effort; and (ii) the refinement may still be in progress and the authors may wish to wait until they can provide a definitive (and therefore error-free) data set. Two further points can be added to this list: (iii) as in every other sphere of life, commercial considerations have recently come into play-access to the coordinates of structures of important disease-related molecules (for example) can be sold to biotechnology companies interested in drug design; and (iv) simply making the time to deposit the coordinates (even with the benefit of electronic submission it can take a day or more to prepare the file for the PDB) may provide a sufficient deterrent.

The biomolecular crystallography community has responded to the problem of deposition through the International Union of Crystallography (IUC) ${ }^{3}$. While recommending that all coordinates be deposited on or before publication, the IUC has included a provision allowing authors to put the coordinates 'on hold' for up to one year after the date of publication. This compromise, scorned by those who advocate immediate release of the data, is accepted by many as the lesser of two evils, in that it satisfies some scientists' commercial or academic interests while at the same time ensuring that the data will be available in a reasonable length of time. And some funding agencies, such as the National Institute of General Medical Sciences in the United States, now make it a condition of 
issuing grants that the investigators deposit coordinates-apparently a very effective means of ensuring deposition. Of course, not all funding agencies have the same requirements.

What role, if any, should the journals that serve the community play? Of necessity, they must respond to the needs and wishes of structural biologists while remaining as objective and non-partisan as possible. So should they also encourage authors to make available their data (coordinates and, to a lesser extent, structure factors-although see ref. 4) available? Because the responsibility for ensuring deposition rests with the entire community and because the scientific journals form an integral part of this community, the answer is surely "yes".

One of the most simple ways of ensuring deposition is to require, as a precondition of publication, that the coordinates be made available. Many journals already require that papers reporting structures be accompanied by data bank accession numbers. What should Nature Structural Biology's stance be? Until now we have encouraged authors to provide a database accession number or an anonymous FTP site address where the coordinates will be available-but have not made this a condition of publication. And all to often we have had to make do with the statement "coordinates will be deposited".

In an effort to promote more prompt deposition we shall require, from January 1996, that authors provide an accession number or an anonymous FTP site address where the coordinates will be generally available. Authors may withhold their coordinates (for further analysis or commercial reasons) for one year from the date of publication (similarly, deposition of structure factors will have a limit of four years), in line with the IUC's provision for delayed release ${ }^{3}$.

Nonetheless, it is our belief that no rule is without exception and we therefore wish to retain editorial discretion regarding the requirement for deposition. In very rare circumstances (as yet unforeseen) we shall be prepared to make exceptions to this precondition for publication-but in such situations it will be incumbent on the authors to provide strong justification for their course of action (with a note in the paper to this effect). Structural biologists who believe in immediate deposition and release will no doubt object to this possible exception, complaining that any less that absolute enforcement will not materially alter the present situation. However, we feel that the strengthening of our requirement for deposition will help to prompt structural biologists who publish in our pages to make their coordinates generally available in a timely fashion.

1. Cohen, J. Science $268,1715-1718$ (1995)

2. Rossman, M.G. \& Argos, P. Acta Crystollagr. B36, 819-823 (1980)

3. International Union of Crystallography Acta Crystollagr. B45, 518-519 (1989)

4. Ten Eyck, L.F. Nature struct. Biol. 2, 1038-1042

\section{ANNOUNCEMENT}

Starting January 1, 1996, data for crystal structures of oligonucleotides should be deposited directly with the Nucleic Acid Database (NDB). Once the data are processed they will be forwarded to the Protein Data Bank (PDB) for prosit in they will be forwarded to the Protein Data Bank (PDB) frop de Protein and make quick be deposited at the PDB. All crystal structure data for DNA and RNA will continue to be available from both the NDB and the PDB.

To deposit the data, submit the coordinates, structure factors and current $P O B$ deposition form to

deposit@ndbserver.rutgers.edu

A preprint of the related material should be mailed or faxed to:

Anke Gelbin,

The Nucleic Acid Database

Department of Chemistry

Rutgers, the State University of New Jersey

P.O. Box 939

Piscataway, New Jersey, USA 08855-0939

Fax: $+1,908.445 .5958$

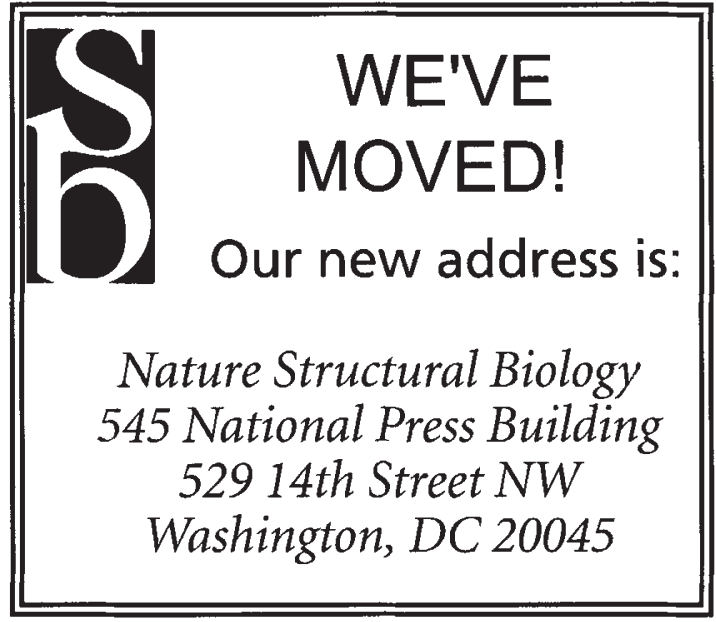

